\title{
Structural characterization and mechanical properties of dextrin-graft-poly(butyl acrylate-co-styrene) copolymers
}

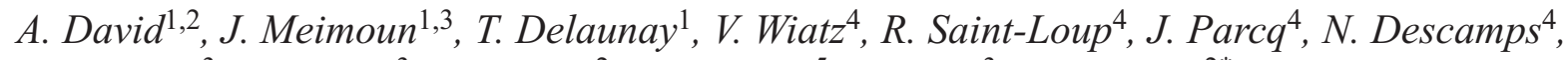 \\ A. Favrelle ${ }^{3}$, F. Bonnet ${ }^{3}$, G. Stoclet ${ }^{2}$, D. Lourdin ${ }^{5}$, P. Zinck ${ }^{3}$, V. Gaucher ${ }^{2}$ \\ ${ }^{1}$ Institut Français des Matériaux Agro-Sourcés (IFMAS), 60 Avenue du Halley, 59650 Villeneuve-d’Ascq, France \\ ${ }^{2}$ Université Lille, Unité Matériaux et Transformations (UMET), CNRS UMR 8207, 59655 Villeneuve d'Ascq, France \\ ${ }^{3}$ Université Lille, Unité de Catalyse et Chimie du Solide (UCCS), CNRS, UMR 8181, 59655 Villeneuve d'Ascq, France \\ ${ }^{4}$ Roquette Frères, 62080 Lestrem Cedex, France \\ ${ }^{5}$ UR1268 Biopolymeres Interactions Assemblage, INRA, F-44300 Nantes, France
}

Received 27 June 2018; accepted in revised form 2 October 2018

\begin{abstract}
Dextrin/starch-graft-poly(butyl acrylate-co-styrene) copolymers have been synthesized by radical graft copolymerization and their structure and mechanical properties are reported. High molecular weight grafted chains formation is favored, leading to a low degree of substitution of starch hydroxyl groups. Wide-Angle-X-ray Scattering (WAXS) analysis indicates that all materials are amorphous and Transmission Electron Microscopy (TEM) investigations reveal a two-phase morphology. This is further confirmed by the presence of two glass transitions, one related to the starch/dextrin macromolecules and sensitive to water content, and one assigned to the grafted polymers that is composition dependent. Water uptake is controlled by dextrin/starch content but diffusivity increases with the butyl acrylate ratio. The mechanical behavior is dependent on monomer ratio, and water content. Increasing butyl acrylate ratio improves the ductility of the sample while materials become brittle as soon as styrene ratio is predominant in the grafted chains. While no effect of molecular weight of starch substrate on structure and thermal behavior is evidenced, dextrin-based materials are slightly more ductile than starchbased ones. The interest of using dextrin instead of starch is further highlighted by a lower viscosity of the reactive medium, together with an improved processability, as structural materials can be obtained over a wider range of composition than with native starch.
\end{abstract}

Keywords: biopolymers, grafted starch, structural organization, water sorption, mechanical behavior

\section{Introduction}

Biobased polymers have received considerable attention over the past recent years, due notably to environmental concerns. Among these, starch is a promising starting material due to its availability and low cost, and thus this polymer has been the subject of numerous studies extensively reviewed [1-5]. However, starch has limited processing ability and exhibits some drawbacks such as a strong hydrophilic character and poor mechanical properties as compared to conventional synthetic polymers [6].
Starch can be plasticized with external plasticizer such as water [7], polyols like glycerol [8], glycols [9], amides [10] or ionic liquids [11] notably. Plasticized starch is used for packaging and agricultural purposes. However, some shortcomings such as plasticizer migration induce an evolution of its properties with time and thus limit its applications. To overcome some of these drawbacks and to achieve desired product properties, the natural polymer can be modified by physical, chemical or enzymatic routes including blending $[12,13]$, chemical functionalization 
such as esterification and etherification $[14,15]$ and graft copolymerization [16]. Graft copolymerization is generally a very versatile way of functionalization providing access to materials displaying a broad combination of properties according to polymers compositions involved $[17,18]$. In particular, the graft copolymerization of vinyl monomers onto starch may lead to starch-based materials with interesting properties [19]. Hydrophobic polymers such as polystyrene and poly(butyl acrylate) can be grafted on the starch backbone. The synthesis of starch-graftpoly(butyl acrylate-co-styrene) was conducted with different radical initiators on native [20], acetylated [21] or oxidized starch [22, 23]. These modifications lead to the formation of starch-based materials displaying improved hydrophobicity and mechanical properties.

Although the synthesis of starch-graft-poly(butyl acrylate- $c o$-styrene) has already been reported in the literature, scarce data are available regarding the structure, thermal and mechanical properties of the resulting materials. Additionally, even if different types of chemically modified starch (native, acetylated, oxidized) were studied, the grafting of butyl acrylate and styrene on a dextrin, i.e. a low molecular weight carbohydrate produced from the hydrolysis of starch, has not been reported to date. The use of dextrin, instead of its native precursor is expected not only to lower the viscosity during the copolymerization process, but also to allow the design of materials displaying original properties.

The goal of this study is to characterize the structure, thermal properties and mechanical behavior of dextrin/starch-graft-poly(butyl acrylate-co-styrene) with various contents of butyl acrylate and styrene. Styrene was chosen for its hydrophobicity and stiffness while butyl acrylate was selected for its flexibility at room temperature. The combination of such monomers in various ratios should allow adjusting properties to the targeted applications. The influence of the weight average molecular weight $\left(M_{\mathrm{w}}\right)$ of the starch substrate on the resulting properties of the materials is also investigated.

\section{Experimental section \\ 2.1. Materials}

Dextrin of maize $\left(M_{\mathrm{w}}=300000 \mathrm{~g} / \mathrm{mol}\right)$ and potato starch were supplied by Roquette Frères (Lestrem, France). Butyl acrylate (99\%) and styrene (99\%) were purchased from Aldrich. Sodium persulfate
(98\%) was purchased from Alfa Aesar. Hydrochloric acid fuming (37\%) was obtained from Merck. Acetone (technical), chloroform (reagent plus) and tetrahydrofuran (analytical grade) were purchased from Fisher. All the reactants and solvents were used as received. Distilled water was used for all experiments.

\subsection{Methods}

\subsubsection{Synthesis of dextrin/}

starch-graft-poly(butyl acrylate-co-styrene)

Dry dextrin $(30 \mathrm{~g})$ or starch $(9 \mathrm{~g})$ was gelatinized in water (in 120 and $140 \mathrm{ml}$ respectively) by heating at $95^{\circ} \mathrm{C}$ for 30 minutes. The temperature was then decreased to $85^{\circ} \mathrm{C}$. Sodium persulfate $(1 \mathrm{~mol} \%$ vs. dextrin/starch), butyl acrylate and/or styrene (100 wt\% $v s$. dextrin/starch) were then added dropwise for 1 hour. The grafting polymerization was carried out for additional 30 minutes. At the end of the reaction, the solution was poured in acetone and left during $24 \mathrm{~h}$ under magnetic stirring. The product was filtered and dried overnight. The ungrafted poly(butyl acrylate-co-styrene) was removed by Soxhlet extraction in chloroform during $24 \mathrm{~h}$.

The reactions with dextrin as the substrate were performed in round-bottom flask reactor whereas the grafting syntheses with native starch were performed in jacketed reactor vessel (anchor blade). The change of reactor was required considering the higher viscosity of starch compared to that of dextrin. In a roundbottom flask, the stirring was not powerful enough to achieve a homogenous medium leading of a grafting percentage below $10 \%$.

The grafting parameters were calculated according to Equations (1) and (2):

Grafting percenta $(G P)[\%]=$

$$
=\frac{\text { weight of grafted polymer }}{\text { weight of dextrin/starch }} \cdot 100
$$

Grafting efficiency $(G E)[\%]=$

$$
=\frac{\text { weight of grafted polymer }}{\text { weight of monomer }} \cdot 100
$$

\subsubsection{Determination of molecular weights of grafted polymers}

Dextrin/starch-graft-poly(butyl acrylate-co-styrene) samples were not soluble in commonly used SEC solvents. Thus, the molecular weights of the grafts were determined by Size Exclusion Chromatography (SEC) after acid hydrolysis according to literature 
procedure [24]. Dextrin/starch-graft-poly(butyl acrylate-co-styrene) $(0.75 \mathrm{~g})$ was stirred in $300 \mathrm{ml}$ of $0.5 \mathrm{M}$ hydrochloric acid for $6 \mathrm{~h}$ under reflux. The resulting polymer was filtered, washed with distilled water and dried in an oven at $30^{\circ} \mathrm{C}$ for $24 \mathrm{~h}$.

The size exclusion chromatography apparatus (SEC, 1260 series, Agilent Technologies) is equipped with Shodex KF-G pre-column and three Shodex columns in series (KF-805, KF-803 et KF-802,5), a Refractive Index Detector and a viscometer calibrated with polystyrene standards in tetrahydrofuran (THF) as the eluent $\left(1 \mathrm{ml} / \mathrm{min}, 40^{\circ} \mathrm{C}\right)$.

\subsubsection{Determination of butyl acrylate/styrene ratio in the grafted chains by ${ }^{1} \mathrm{H}$ Nuclear Magnetic Resonnance (NMR) analysis}

Dextrin/starch-graft-poly(butyl acrylate-co-styrene) copolymers were not soluble in deuterated Dimethylsulfoxide (DMSO- $\mathrm{d}_{6}$ ) as soon as the styrene ratio in the grafted statistical copolymer exceeded $40 \%$. The ${ }^{1} \mathrm{H}$ NMR analyses were thus performed on grafted chains obtained after hydrolysis of dextrin/ starch-graft-copolymers (Figure 1). The grafted chains are indeed soluble in a mixture of $\mathrm{DMSO}-\mathrm{d}_{6} /$ Chloroform $\left(\mathrm{CDCl}_{3}\right)(50 / 50)$ after stirring for a few hours at $80^{\circ} \mathrm{C}$. The advantage of carrying out the NMR analysis in this solvent composition is to shift the chloroform signal from 7.26 to $8 \mathrm{ppm}$, thus avoiding the superposition of the aromatic signals of styrene with that of chloroform.

${ }^{1} \mathrm{H}$ NMR spectra of the grafted chains recovered after hydrolysis (4-5 mg) were recorded on Bruker Avance $400 \mathrm{MHz}$ instrument (delay time $=4 \mathrm{~s}$ and number of scan $=32$ ) at room temperature in a mixture of DMSO-d $\mathrm{d}_{6} / \mathrm{CDCl}_{3}(50 / 50,0.5 \mathrm{ml})$ as the solvent. The chemical shifts were calibrated using the residual signal of DMSO- $\mathrm{d}_{6}$.

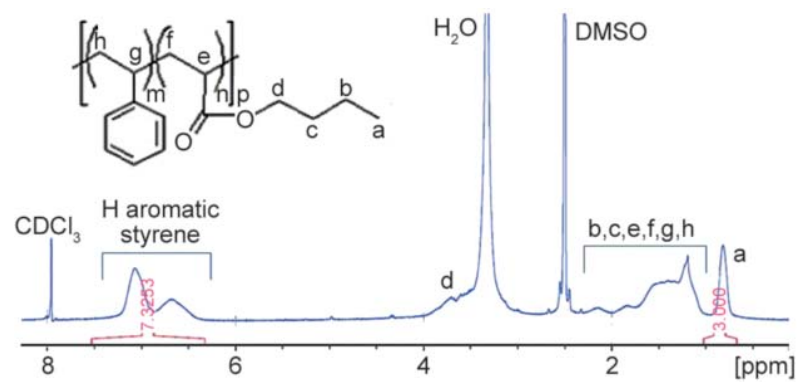

Figure 1. ${ }^{1} \mathrm{H}$ NMR spectrum of the grafted chains of poly (butyl acrylate-co-styrene) obtained after acid hydrolysis. Solvent: 50/50 mixture of DMSO- $\mathrm{d}_{6} /$ $\mathrm{CDCl}_{3}(400 \mathrm{MHz})$.
The following method was applied to determinate the butyl acrylate/styrene ratio in the grafted chains. First, the signal between 0.7 and $1 \mathrm{ppm}$ was chosen as the calibration base: $I_{0.7-1} \mathrm{ppm}=3 \mathrm{H}\left(\mathrm{CH}_{3}\right.$ corresponding to butyl group of the butyl acrylate unit). The signal between 6.4 and $7.6 \mathrm{ppm}$ was then integrated and assigned to the 5 aromatics protons of the styrene unit. The grafting percentages for the butyl acrylate (BA) and styrene (St) units were obtained according to Equations (3) to (6):

Mass ratios:

$r_{\mathrm{BA}}=\frac{X_{1 \mathrm{BA}}}{X_{1 \mathrm{BA}}+X_{2 \mathrm{St}}} \cdot 100$

$r_{\mathrm{St}}=\frac{X_{2 \mathrm{St}}}{X_{1 \mathrm{BA}}+X_{2 \mathrm{St}}} \cdot 100$

where

$X_{1 \mathrm{BA}}=\frac{I_{0.7-1 \mathrm{ppm}}}{3} \cdot M_{\mathrm{BA}}$

$X_{2 \mathrm{St}}=\frac{I_{6.4-7.6 \mathrm{ppm}}}{5} \cdot M_{\mathrm{St}}$

\subsubsection{Structural and morphological characterizations}

$200 \mu \mathrm{m}$ thick films were prepared by compression molding $\left(P=200\right.$ bars, $\left.T=165^{\circ} \mathrm{C}, t=10 \mathrm{~min}\right)$. Prior to any characterization, all films were stored at $20^{\circ} \mathrm{C}$ under $57 \%$ relative humidity $(R H)$ for one week in order to reach moisture content equilibrium.

Wide-Angle-X-ray Scattering (WAXS) experiments were carried out on a Genix microsource (XENOCS) equipment using the $\mathrm{Cu} \mathrm{K}_{\alpha}$ radiation $(\lambda=1.54 \AA)$. Primary beam was collimated and monochromatized by a FOX2D-12Inf optic (Xenocs, France), and the WAXS patterns were collected on a CCD VHR detector (Photonic Sciences). The experiments were conducted at room temperature in transmission mode on the compression molded films. Standard corrections were applied to the WAXS patterns before their treatments. The intensity profiles were obtained by $360^{\circ}$ azimuthal integration of the 2D WAXS patterns using the fit2D software ${ }^{\circledR}$.

Transmission Electron Microscopy (TEM) was performed on a Philips CM120 operated at $120 \mathrm{kV}$ (CTM, Lyon, France). Ultrathin slices $(\sim 70 \mathrm{~nm})$ were cut using an Ultra-cut cryo-microtome. Temperature of the diamond knife was fixed at room temperature 
and $-65^{\circ} \mathrm{C}$ for the dextrin-graft-poly(styrene) and dextrin-graft-poly(butyle acrylate) films, respectively. The slices were then stained by exposure to the vapor of Ruthenium tetroxide $\left(\mathrm{RuO}_{4}\right)$ for the dextrin-graft-poly(styrene) sample and of Osmium tetroxide $\left(\mathrm{OsO}_{4}\right)$ for the dextrin-graft-poly(butyle acrylate) sample in order to achieve contrast of the grafted chain domains from the dextrin phase.

\subsubsection{Thermal analysis}

Thermal properties were determined by means of Differential Scanning Calorimetry (DSC) experiments carried out on a DSC Q2000 (Thermal Analysis) instrument calibrated with a high purity indium sample according to standard procedures. The samples of approximately $15 \mathrm{mg}$ were put in sealed pans and analyzed in the temperature range from -90 to $200^{\circ} \mathrm{C}$ at a heating rate of $10^{\circ} \mathrm{C} / \mathrm{min}$ under nitrogen atmosphere.

\subsubsection{Water vapor uptake and diffusivity}

Water vapor uptake was determined at room temperature using TA Instruments Q5000 Dynamical Vapor Sorption (DVS). Before each analysis, samples were dried at $60^{\circ} \mathrm{C}$ and $0 \%$ Relative Humidity $(R H)$ until their weight was stable (relative weight variation $\Delta w<10^{-2} \%$ for $15 \mathrm{~min}$ ). Samples were then stabilized at $20^{\circ} \mathrm{C}$ under various moisture conditions (30, 50 and $80 \% R H$ ) with the same equilibrium criterion $\left(\Delta w<10^{-2} \%\right.$ for $\left.15 \mathrm{~min}\right)$.

During vapor sorption at constant temperature, we considered that the process follows the second Fick's law. Taking into account sample geometry (thickness $\sim 0.1$ width), the case of one-dimensional diffusion is assumed (water molecules penetrate through thickness and a negligible amount through the edges) so that the second Fick's law (Equation (7)) may be expressed as follows:

$$
\frac{\partial C}{\partial t}=D \frac{\partial^{2} C}{\partial x^{2}}
$$

where $C$ is the moisture content at a specified time $t$, and $x$ the direction of diffusion.

The details of the solution of this equation as well as the determination of the diffusion coefficient from the experimental data are already reported in literature. To summarize, Fickian diffusion is expressed according to Equation (8):

$\frac{C(t)-C_{0}}{C_{\infty}-C_{0}}=\frac{M_{t}}{M_{\infty}}=\frac{4}{\sqrt{\pi}} \sqrt{\frac{D t}{e}}$

where $C_{0}$ the initial concentration of water, $C(t)$ the concentration at time $t, C_{\infty}$ the concentration at equilibrium, $M(t)$ and $M_{\infty}$ the mass at time $\mathrm{t}$ and at the equilibrium state respectively, $D$ the diffusion coefficient, and $e$ the thickness of the film. At short times, Equation (8) provides a linear dependence of the normalized mass intake $M(t) / M \infty$ as a function of $\sqrt{t}$ and the diffusion coefficient $D$ is then determined experimentally from the slope of this curve.

\subsubsection{Uniaxial tensile behavior}

Mechanical behavior was studied in uniaxial tensile mode using an Instron 4466 apparatus. Experiments were performed at an initial strain rate of $10^{-2} \mathrm{~s}^{-1}$ at room temperature under various moisture contents. Specimens with gauge width and length $5 \mathrm{~mm} \times$ $24 \mathrm{~mm}$ were cut off the dextrin/starch copolymers films by laser cutting.

\section{Results and discussion}

\subsection{Synthesis of dextrin/starch-graft- poly(butyl acrylate-co-styrene)}

The graft copolymerization reaction scheme is represented on Figure 2. The mechanism of free radical polymerization using sodium persulfate as radical

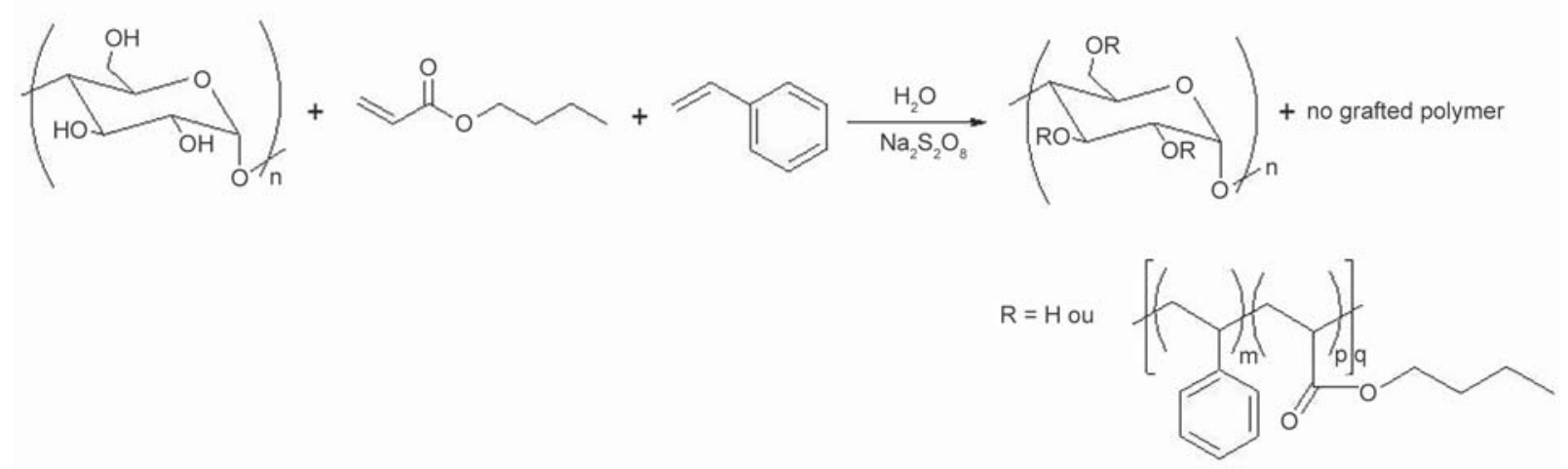

Figure 2. Grafting of poly(acrylate butyle-co-styrene) on dextrin/starch. 
initiator can be divided in three steps: initiation, propagation and termination that are presented in Figure 3 [24]. During the initiation step, radicals are formed on dextrin by hydrogen transfer to the initiator. In aqueous medium, persulfate decomposes into several radical species upon heating able to react with dextrin. Dextrin radicals react further with the double bond of the vinyl monomer (styrene or butyl acrylate), leading to a dextrin-grafted monomer radical that induces polymerization. Termination reaction can occur by combination or disproportionation of two reactive chains. Similar mechanism is operating in the case of starch.

One of the objectives of the study is to modify starch or dextrin, while maintaining a mainly biobased material. The $50 / 50 \mathrm{wt} \%$ of dextrin or starch/monomers fraction was the higher biopolymer ratio leading to suitable material. Entries representative of the grafting reactions of poly(butyl acrylate), polystyrene or poly (butyl acrylate-co-styrene) copolymers onto starch or dextrin, starting from a 50/50 $\mathrm{wt} \%$ of dextrin or starch/monomers ratio, are presented in Table 1 . The grafting percentages obtained are high $(G P=72$ $97 \%$ ) and quite similar regardless of the nature of the substrate, except for the grafting of polystyrene onto native starch. Formation of the ungrafted copolymer is not favored in the medium. However, one may note that the grafting percentages tend to decrease on all substrates when styrene content is larger than its acrylate counterpart. This phenomenon may be attributed to the slower polymerization kinetics resulting from the lower solubility of styrene vs. butyl acrylate in water. Regarding the reactions conducted with styrene as the only monomer in the medium, a drop in the grafting percentage on starch substrate down to $G P=$ $48 \%$ was observed. Two phases were observed after 30 minutes of reaction: an aqueous phase in which the polymer was grafted onto starch and a hydrophobic

Initiation step

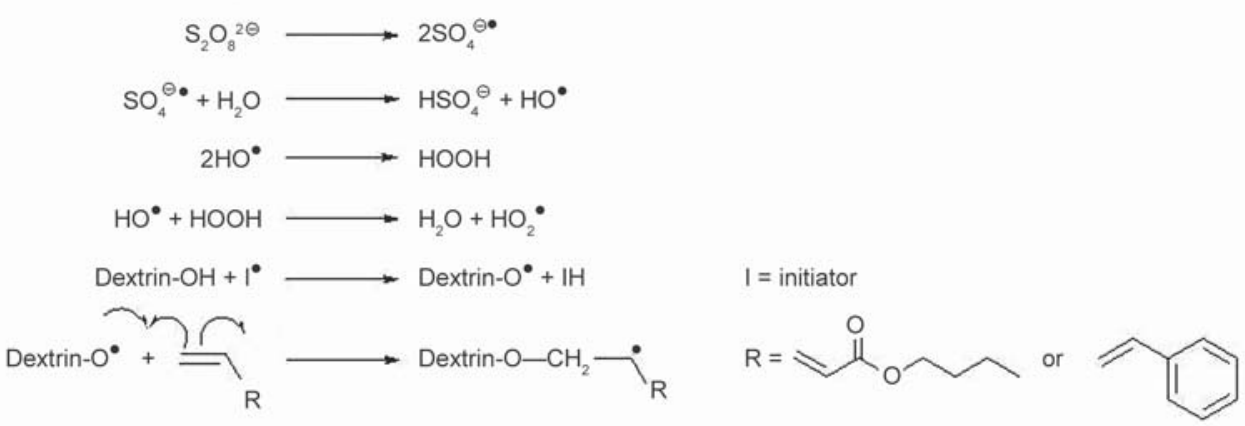

Propagation

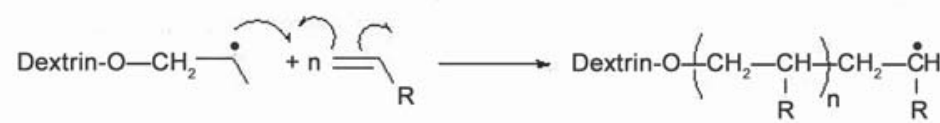

Terminaison

graft terminaison by coupling with initiator radical

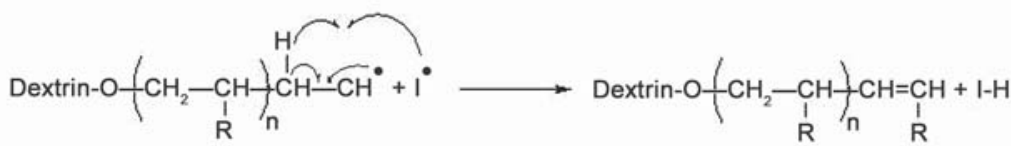

graft terminaison by coupling or combination leading eventually to crosslinking

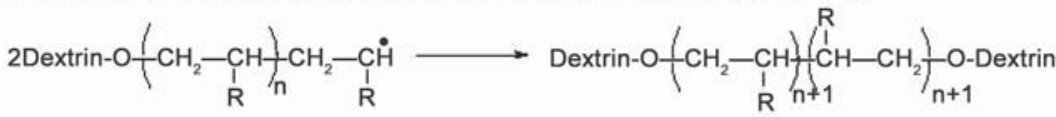

graft terminaison by disproportionation

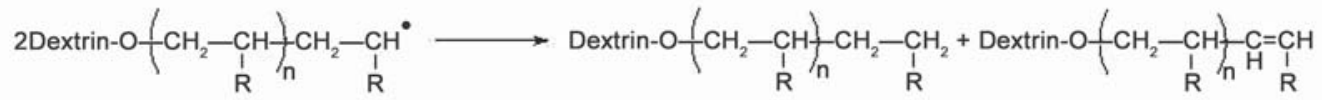

Figure 3. Mechanism of grafting by free radical polymerization. 
Table 1. Grafting results and molar average molecular weight of dextrin/starch-graft-poly(butyl acrylate-co-styrene) ${ }^{\mathrm{a}}$.

\begin{tabular}{|c|c|c|c|c|c|c|}
\hline Substrate & \begin{tabular}{|c|}
$\% \mathrm{BA} / \% \mathrm{St}$ \\
(introduced in the feed)
\end{tabular} & Sample name & $\begin{array}{c}G P_{\text {total }} \text { b } \\
{[\%]}\end{array}$ & $\begin{array}{l}\text { Weight ratio of } \mathrm{BA} / \mathrm{St} \\
\text { units in grafted chains }\end{array}$ & $\begin{array}{l}M_{\mathrm{n} \text { grafted chains }}{ }^{\mathrm{d}} \\
{[\mathrm{g} / \mathrm{mol}]}\end{array}$ & $\boldsymbol{\nexists}^{\mathbf{e}}$ \\
\hline \multirow{5}{*}{$\begin{array}{l}\text { Dextrin } \\
\left(M_{\mathrm{W}}=300000 \mathrm{~g} / \mathrm{mol}\right)\end{array}$} & $100 / 0$ & D- $g$-PBA & 97 & $100 / 0$ & \multicolumn{2}{|c|}{ Insoluble in THF } \\
\hline & $75 / 25$ & $\mathrm{D}-g-\mathrm{P}(\mathrm{BA}-c o-\mathrm{St} 75 / 25)$ & 88 & $75 / 25$ & 168100 & 6.2 \\
\hline & $50 / 50$ & D- $g$-P(BA-co-St 50/50) & 72 & $49 / 51$ & 125200 & 3.6 \\
\hline & $25 / 75$ & D-g-P(BA-co-St 25/75) & 74 & $23 / 77$ & 162000 & 3.0 \\
\hline & $0 / 100$ & D-g-PS & 91 & $0 / 100$ & 123300 & 3.5 \\
\hline \multirow{5}{*}{$\begin{array}{l}\text { Native starch } \\
\left(M_{\mathrm{w}}=10^{6}-10^{7} \mathrm{~g} / \mathrm{mol}\right)\end{array}$} & $100 / 0$ & S- $g$-PBA & 86 & $100 / 0$ & \multicolumn{2}{|c|}{ Insoluble in THF } \\
\hline & $75 / 25$ & S-g-P(BA-co-St 75/25) & 97 & $75 / 25$ & 74390 & 9.8 \\
\hline & $50 / 50$ & S- $g-\mathrm{P}(\mathrm{BA}-c o-\mathrm{St} 50 / 50)$ & 91 & $49 / 51$ & 51300 & 4.7 \\
\hline & $25 / 75$ & S- $g$-P(BA-co-St 25/75) & 77 & $27 / 73$ & 41620 & 3.1 \\
\hline & $0 / 100$ & S-g-PS & 48 & $0 / 100$ & 38780 & 7.6 \\
\hline
\end{tabular}

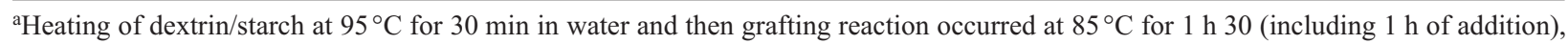
sodium persulfate ( $1 \mathrm{~mol} \% \mathrm{vs}$. dextrin/starch)

${ }^{b}$ grafting percentages were calculated by gravimetry

${ }^{c}$ ratios of BA or St units in grafted chains were determined by ${ }^{1} \mathrm{H}$ NMR analysis in DMSO- $\mathrm{d}_{6} / \mathrm{CDCl}_{3}(50 / 50)$ (cf. § 2.2.3.)

${ }^{\mathrm{d}} M_{\mathrm{n}}$ of grafted chains were determined by SEC $\left(40^{\circ} \mathrm{C}\right.$, THF, PS standards $)$ after acid hydrolysis

${ }^{\mathrm{e}} \oslash=M_{\mathrm{w}} / M_{\mathrm{n}}$

one in which the homopolymer was formed and which was fed with styrene during the addition of the monomer.

The solubility of the dextrin/starch-graft-poly(butyl acrylate-co-styrene) copolymers depends on the composition of the grafts. They were found to be insoluble in common NMR solvents for styrene/acrylate ratios greater than $40 \%$. A typical ${ }^{1} \mathrm{H}$ NMR spectrum is presented in Figure 4. The signals characteristic of the dextrin can be found between 3 and $6 \mathrm{ppm}$, with notably the anomeric proton at $5 \mathrm{ppm}$. Signals representative of the synthetic part can also be detected, notably the aromatic protons of styrene between 6.5 and $7.5 \mathrm{ppm}$ and the $-\mathrm{CH}_{3}$ of butyl acrylate around $0.7 \mathrm{ppm}$. Due to the insolubility of several dextrin/ starch-graft-poly(butyl acrylate-co-styrene) copolymers, the composition of the grafts was determined after hydrolysis, as reported in the experimental part (§ 2.2.3.). It can be seen that the composition of the graft follows the monomer feed.

$M_{\mathrm{n}}$ of the grafted chains determined by SEC display values ranging from $3.8 \cdot 10^{4}$ to $7.4 \cdot 10^{4}$ and $1.2 \cdot 10^{5}$ to $1.6 \cdot 10^{5} \mathrm{~g} / \mathrm{mol}$ (Table 1 ) in the case of starch and dextrin respectively. From a structural point of view, these results indicate that $M_{\mathrm{w}}$ of the grafted chains is almost of the same order of magnitude ( $\times 1.5$ to 3.4$)$ as that of the dextrin $\left(M_{\mathrm{w}}=300000 \mathrm{~g} / \mathrm{mol}\right)$ but lower in the case of starch $\left(M_{\mathrm{W}}=10^{6}-10^{7} \mathrm{~g} / \mathrm{mol}\right.$ typically). The differences observed on molecular weight values could be due in part to the fact that the reactions are not carried out in the same reactor for the two kinds of substrates (cf. experimental part). The influence of the butyl acrylate/styrene $(\mathrm{BA} / \mathrm{St})$ ratio on the molecular weights of the grafted chains has to be considered

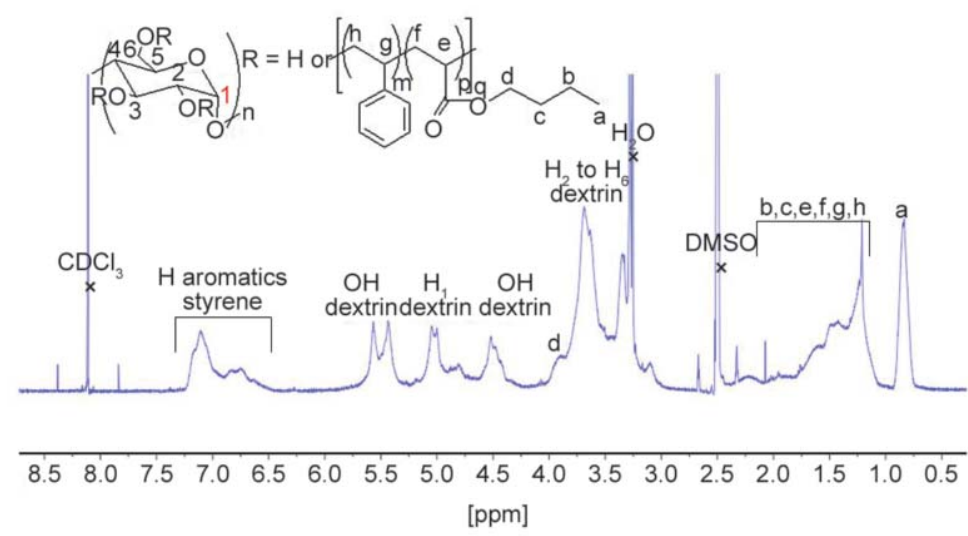

Figure 4. ${ }^{1} \mathrm{H}$ NMR spectrum of dextrin-graft-poly(butyl acrylate-co-styrene) $(\mathrm{BA} / \mathrm{St}=75 / 25)$, in a 50/50 mixture of DMSO- $\mathrm{d}_{6} /$ $\mathrm{CDCl}_{3}$. 
Table 2. Degree of substitution and average number of glucose units between two grafted chains of dextrin/starch-graftpolystyrene copolymers.

\begin{tabular}{|l|c|c|c|c|c|}
\hline \multicolumn{1}{|c|}{ Substrate } & $\begin{array}{c}\boldsymbol{G P} \\
{[\mathbf{\%}]}\end{array}$ & $\begin{array}{c}\boldsymbol{M}_{\mathbf{n}}^{\mathbf{a}} \\
{[\mathbf{g} / \mathbf{m o l}]}\end{array}$ & $\boldsymbol{D P}_{\mathbf{n}}{ }^{\mathbf{b}}$ & $\boldsymbol{D S}\left(\cdot \mathbf{1 0}^{\mathbf{- 3}}\right)^{\mathbf{c}}$ & $\begin{array}{c}\text { Average number of glucose units } \\
\text { between two grafted chains }^{\mathbf{d}}\end{array}$ \\
\hline Dextrin & 91 & 123300 & 1185 & 1.2 & 834 \\
\hline Native starch & 48 & 38780 & 372 & 2.0 & 497 \\
\hline
\end{tabular}

${ }^{\mathrm{a}} M_{\mathrm{n}}$ determined by SEC $\left(40^{\circ} \mathrm{C}\right.$, THF, PS standards)

${ }^{\mathrm{b}} D P=M_{\mathrm{n}} / M_{\mathrm{St}}$ with $M_{\mathrm{St}}:$ molar weight of styrene unit

${ }^{\mathrm{c}} D S=$ number of grafted chains per glucose units $=\left(n_{\text {molar St units }} / D P_{\mathrm{n}}\right) / n_{\text {molar glucose units }}$ with $n_{\text {molar glucose units }}=m_{\text {starch }} / M_{\text {glucose }}$ and $n_{\text {molar St units }}=m_{\text {St units }} / M S t=\left[(G P[\%] / 100) \cdot m_{\text {starch }}\right] / M_{\text {St }}$

${ }^{\mathrm{d}}$ Average number of glucose units between two grafted chains $=\left[n_{\text {molar glucose units }} /\left(n_{\text {molar St units }} / D P_{\mathrm{n}}\right)\right]^{-1}$

with caution, as molecular weights are determined using polystyrene standards. It also appears that the dispersities are very broad $(D=3-10)$.

Regarding macromolecular structure, the formation of a few long grafted chains is favored at the expenses of numerous short grafted chain developments. The determination of the exact $M_{\mathrm{n}}$ value is only possible for dextrin/starch-graft-polystyrene copolymers due to the fact that the polymer grafted has the same chemical nature as the SEC standard (Table 2). A very low degree of substitution $(D S)$ of dextrin/ starch $\mathrm{OH}$ functions $(D S<0.002)$ is obtained for dextrin/starch-graft-polystyrene, which corresponds to an average of ca. 500 to 800 glucose units between two grafts. The low DS can be at least partially linked to the amount of initiator introduced in the reaction medium. For $1 \mathrm{~mol} \%$ initiator $v s$. dextrin/starch, the maximum value of degree of substitution is around
0.01 considering a theoretical quantitative decomposition of the initiator.

\subsection{Structural analysis of dextrin/starch- graft-poly(butyl acrylate-co-styrene)}

Structural characterization of starch-graft-poly(butyl acrylate-co-styrene) materials as well as of the reference native starch is reported in Figure 5. The WAXS profiles of pure polystyrene (PS) and pure poly(butyl acrylate) (PBA) are also reported for the sake of comparison.

By contrast to the native potato starch which displays a B-type semi-crystalline structure (dotted brown curve), the gelatinized starch exhibits an amorphous structure as revealed by the broad halo centered around $2 \theta=20^{\circ}$ (black curve). This result shows that the thermal treatment (starch in excess water heated at $95^{\circ} \mathrm{C}$ for $30 \mathrm{~min}$ ) was sufficient to completely

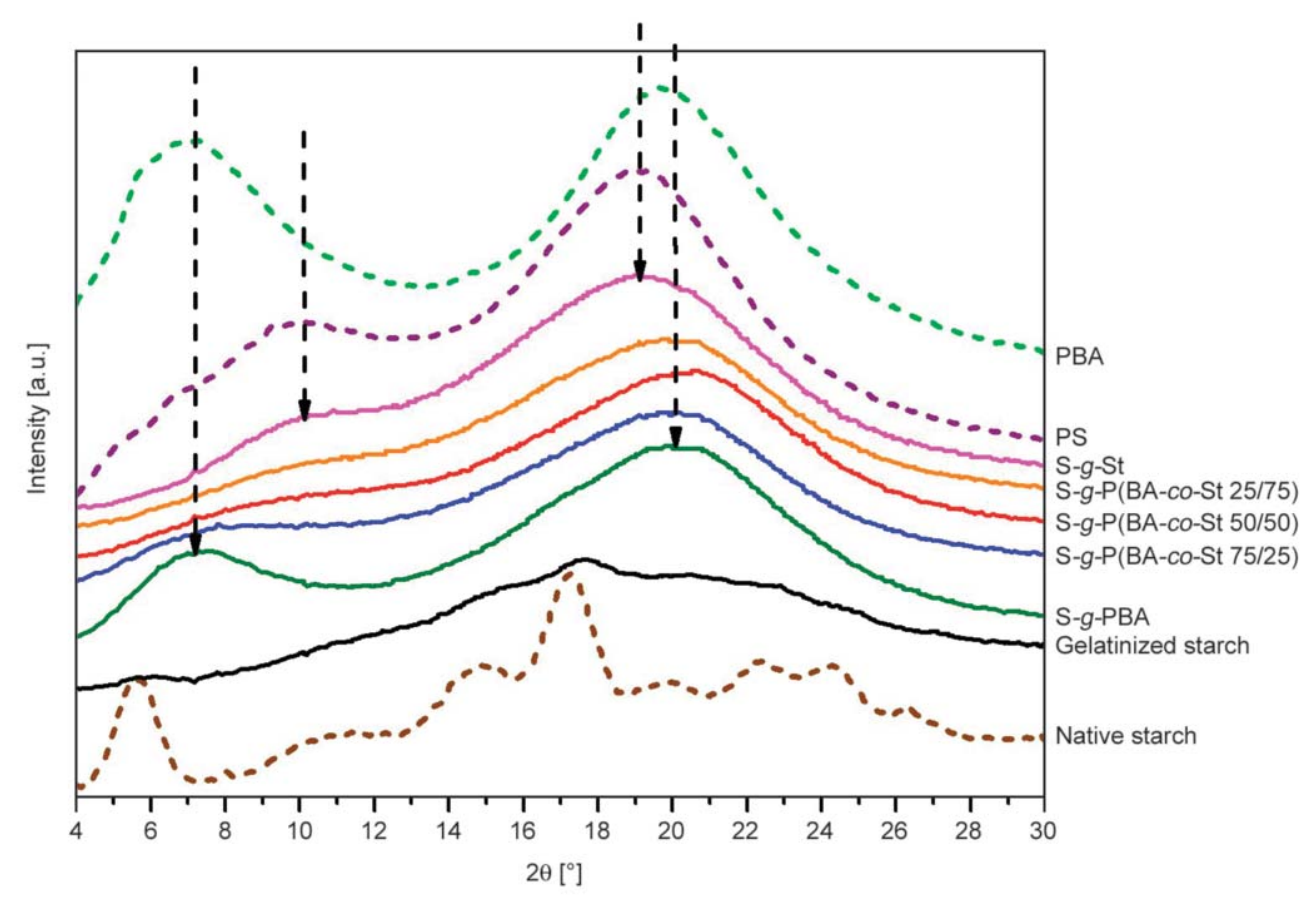

Figure 5. WAXS profiles of native and gelatinized starch and starch-graft-poly(butyl acrylate-co-styrene) films. 
destroy the crystalline structure of the native starch and that no retrogradation has occurred during the cooling and film elaboration steps. All starch-graftpoly(butyl acrylate-co-styrene) samples are fully amorphous, as evidenced by the broad halo towards $2 \theta=20^{\circ}$ whatever the $\mathrm{BA} / \mathrm{St}$ ratio. Note that an additional broad peak is observed around $2 \theta=7$ and $10^{\circ}$ especially in the case of samples with $100 \%$ butyl acrylate and $100 \%$ styrene contents, respectively. These observations indicate the presence of an additional characteristic length in the materials. Several authors which have already reported this kind of diffractograms for polystyrene $[25,26]$, poly(acrylate) and poly(alkyl methacrylate) $[27,28]$ attribute the origin of this additional peak to a scattering signal related to the lateral groups of the same size and almost regularly spaced.

To summarize, the main amorphous halo located around $20^{\circ}$ results from the contribution of both the gelatinized starch and the grafted chains, while the second scattering peak at lower Bragg angle is related to the packing of the aromatic groups in the case of polystyrene and of the ester groups in the case of poly(butyl acrylate). The disappearance or decrease in intensity of this peak for the intermediate BA/St ratios indicates a loss of this characteristic length, which may suggest that the grafted chains are rather composed of random copolymers.

Similar trends are observed for dextrin-graft-poly (butyl acrylate-co-styrene) copolymers revealing that molecular weight of the substrate has no major impact on the molecular organization of materials.

\subsection{Thermal properties and morphology}

As presented in Figure 6a, thermograms of the dextrin-graft-poly(butyl acrylate-co-styrene) samples stored at $20^{\circ} \mathrm{C}$ and $50 \% R H$ exhibit two glass transitions $\left(T_{\mathrm{g}}\right)$ :

- The first one $\left(T_{\mathrm{g} 1}\right)$ is located around $55 \pm 3{ }^{\circ} \mathrm{C}$, whatever the composition of the grafts. For the sake of comparison, gelatinized dextrin sample stored under the same conditions displays a $T_{\mathrm{g}}$ in the same temperature range which suggests that $T_{\mathrm{g} 1}$ is related to the dextrin backbones.

- The second one $\left(T_{\mathrm{g} 2}\right)$ occurs between -44 and $100^{\circ} \mathrm{C}$ depending on the composition of the grafts. In particular, the $T_{\mathrm{g} 2}$ value increases with the amount of styrene. This thermal event may be attributed to the grafted chains. Note that a good agreement is obtained between the measured value of $T_{\mathrm{g} 2}$ and the calculated one from a simple law of mixture considering the weight ratio of styrene and butyl acrylate monomers in the grafted chains and taking a $T_{\mathrm{g}}$ value of -44 and $100^{\circ} \mathrm{C}$ for the corresponding homopolymers. This is in agreement with a statistical microstructure, as suggested by the previous X-ray diffraction study and by the values of the reactivity ratios of the comonomers (styrene (1)/butyl acrylate (2) $r_{1}=k_{11} / k_{12}=0.7-$ 0.9 and $\left.r_{2}=k_{22} / k_{12}=0.17-0.30\right)$ [29]. Care has to be taken however, as the experimental conditions of [29] differ from ours.

In order to reinforce these findings, DSC experiments were conducted on samples stored under $20^{\circ} \mathrm{C}$ and $80 \% R H$. As can be seen in Figure $6 \mathrm{~b}, T_{\mathrm{g} 1}$ decreases from 55 to $-8^{\circ} \mathrm{C}$ as for the pure gelatinized dextrin while $T_{\mathrm{g} 2}$ remains unchanged. These observations are in good agreement with the assignment of the glass transitions previously proposed. The grafted chains composed of hydrophobic groups are thus insensitive to moisture while the decrease of $T_{\mathrm{g} 1}$ is related to the plasticization effect of water on the dextrin chains due to the higher amount of water sorbed at higher relative humidity.

Starch-graft-poly(butyl acrylate-co-styrene) materials exhibit similar thermal behavior: two glass transitions are observed in the same temperature range as in the case of dextrin-based materials stored under the same conditions.

Observation of two glass transitions is an indication of a phase separation. According to Utracki [30], distinct glass transitions may be observed as soon as the domain size exceeds $30 \mathrm{~nm}$. Observations by transmission electron microscopy were carried out on dextrin-graft-poly(butyl acrylate) and dextrin-graftpolystyrene samples and images are reported on Figure $6 \mathrm{c}$ and Figure $6 \mathrm{~d}$. Two co-continuous phases are evidenced whatever the nature of the grafted chain. These observations are quite consistent with the previous results obtained by thermal analysis. It is worth mentioning that the sizes of the butyl acrylate chains rich domains are smaller $(\approx 50 \mathrm{~nm})$ than the ones related to polystyrene rich domains $(\approx 200 \mathrm{~nm})$. Further investigations are necessary to determine the origin of this phenomenon.

\subsection{Water uptake and diffusivity as a function of grafted chain composition}

Water sorption and diffusivity data of the dextrin-graftpoly(butyl acrylate-co-styrene) films are gathered in 

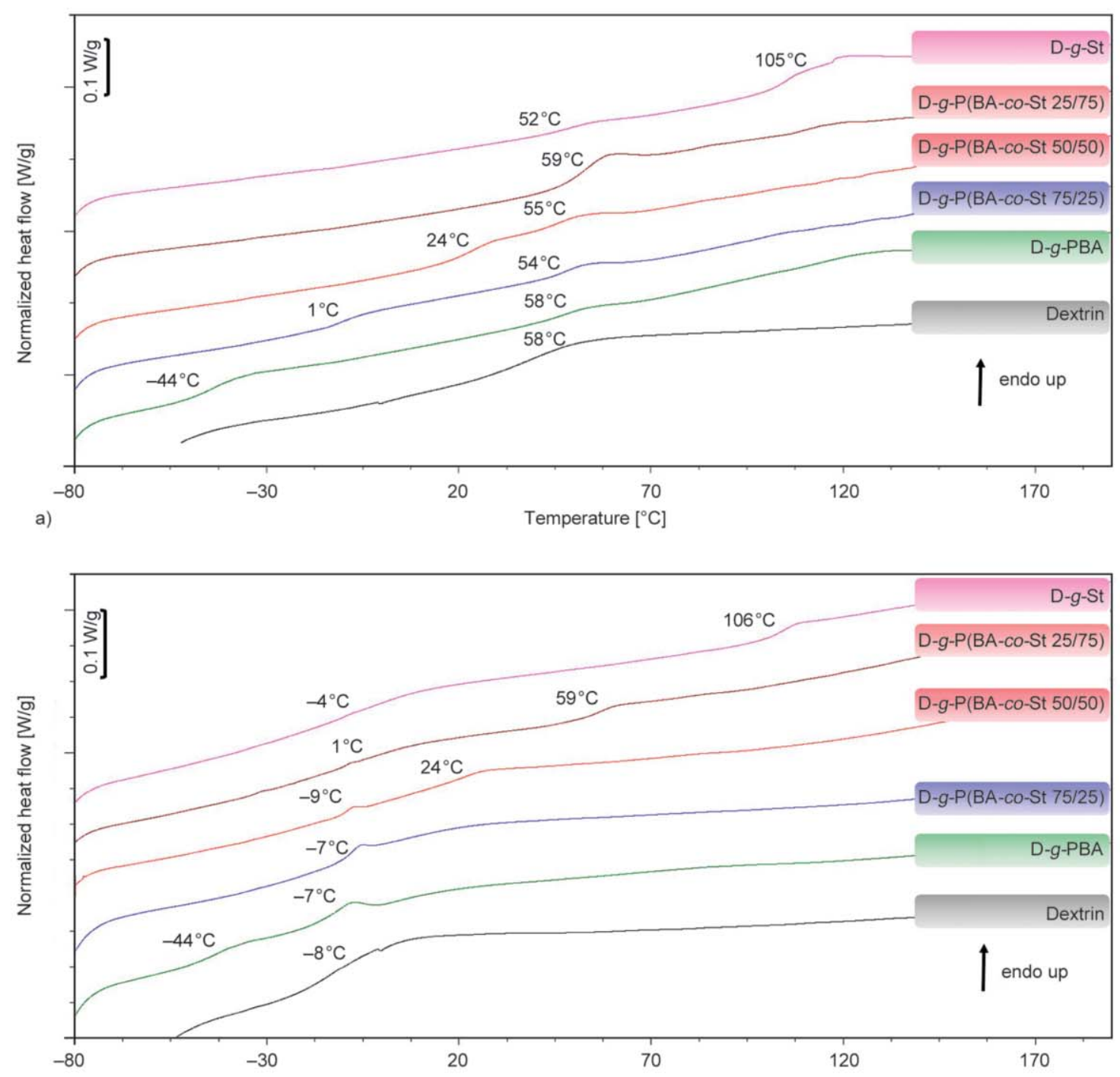

b)

Temperature $\left[{ }^{\circ} \mathrm{C}\right]$

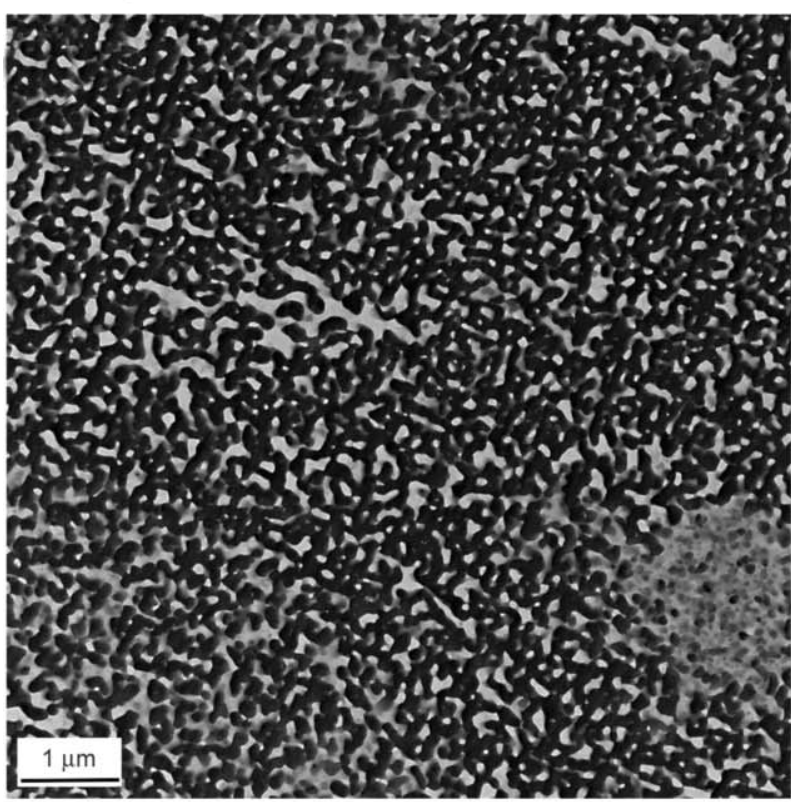

c)

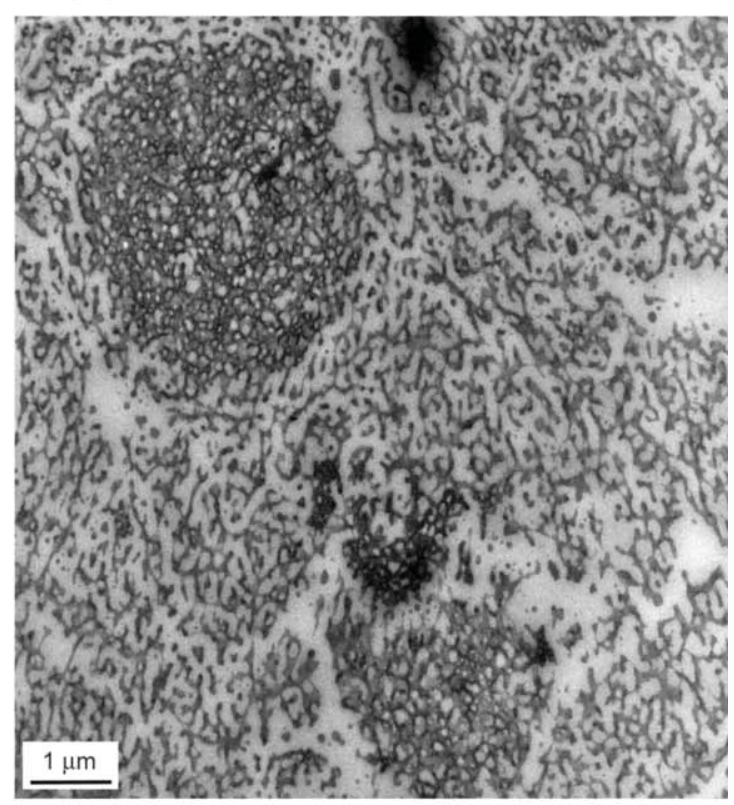

d)

Figure 6. DSC curves of gelatinized dextrin and dextrin-graft-poly(butyl acrylate-co-styrene) samples stored under $20^{\circ} \mathrm{C}$ and a) $50 \% R H$ and b) $80 \% R H$ and TEM micrographs of c) dextrin-graft-polystyrene d) dextrin-graft-poly(butyl acrylate). 
Table 3. Water uptake of sample at room temperature as a function of the relative humidity, and water diffusion coefficient $D$ at $50 \% R H$ and $20^{\circ} \mathrm{C}$ (values between brackets represent the water uptake by weight of dextrin).

\begin{tabular}{|c|c|c|c|c|}
\hline Material & $30 \% R H$ & $50 \% R H$ & $80 \% R H$ & $\begin{array}{l}D \cdot 10^{-14} \\
{\left[\mathrm{~m}^{2} \cdot \mathrm{s}^{-1}\right]} \\
50 \% R H\end{array}$ \\
\hline Dextrin & 5.2 & 8.4 & 14.7 & 0.06 \\
\hline D-g-PBA & $3.1(6.2)$ & $4.6 \quad(9.2)$ & $8.2(16.4)$ & 0.91 \\
\hline D-g-P(BA-co-St 75/25) & $3.2(6.4)$ & $4.8 \quad(9.6)$ & $8.5(17.0)$ & 0.35 \\
\hline $\mathrm{D}-g-\mathrm{P}(\mathrm{BA}-c o-\mathrm{St} 50 / 50)$ & $3.5(7.0)$ & $5.3(10.6)$ & $8.7(17.4)$ & 0.21 \\
\hline D-g-P(BA-co-St 25/75) & $3.3(6.6)$ & $5.1(10.2)$ & $8.1(16.2)$ & 0.15 \\
\hline $\mathrm{D}-g$-PS & $2.9(5.8)$ & $4.0 \quad(8.0)$ & $6.5(13.0)$ & 0.11 \\
\hline
\end{tabular}

Table 3. The values between brackets correspond to the water uptake per weight fraction of dextrin in the sample. Dextrin-graft-poly(butyl acrylate-co-styrene) films sorb lower water content than pure dextrin sample and as expected, the water sorbed by the films increases with relative humidity. However, as shown in Table 3, the amount of water sorbed normalized by weight fraction of dextrin is similar to that of pure dextrin, regardless of the composition. This result is not surprising considering that grafted chains are rather hydrophobic and that the substitution degree is very low. The number of sorption sites $(-\mathrm{OH}$ groups) per $\alpha$-D-glucose cycle may be considered to be equal to three, as in pure dextrin. It seems that the amount of water sorbed in dextrin-graft-poly(butyl acrylate-co-styrene) films is controlled by the dextrin weight fraction. By contrast, the composition of the grafted chain has an important impact on the water diffusivity. As shown in Table 3, the diffusion coefficient $D$ gradually decreases with the BA/St ratio of the grafted chain. For example, $D$ decreases by a factor 10 between dextrin-graft-poly(butyl acrylate) and dextrin-graft-polystyrene samples. This result may be related to the $T_{\mathrm{g}}$ of the grafted chains, since the poly(butyl acrylate) and polystyrene have a glass transition temperature of about -44 and $100^{\circ} \mathrm{C}$ respectively. This indicates that the more flexible the grafted chain, the easier the water diffusion is.

Note that the diffusion coefficient value for dextrin, which is of the same order of magnitude as the one reported in literature for starch [31], is low compared to those of dextrin-graft-(butyl acrylate-co-styrene) materials. The glass transition of polystyrene being higher than the one of dextrin stored under ambient conditions, this shows that others parameters play a role in diffusivity. In particular, it may be suspected that there is lower amount of free volume for pure dextrin due to more compact H-bond network compared to dextrin-graft-(butyl acrylate-co-styrene).
Further investigations are necessary to better understand this behavior.

Starch-graft-poly(butyl acrylate-co-styrene) samples display similar behavior. This result may be not surprising considering that the amount of water sorbed is rather controlled by the starch phase and that diffusivity is mainly influenced by the glass transition of the (butyl acrylate-co-styrene) phase.

\subsection{Mechanical behavior as a function of grafted chain composition and relative humidity}

Nominal stress-strain curves of dextrin-graft-poly (butyl acrylate- $c o$-styrene) films uniaxially drawn at $20^{\circ} \mathrm{C}$ and $50 \% R H$ are presented in Figure 7. Similar results are obtained with starch-based samples. Note however that it was not possible to produce tensile specimens in the case of starch-graft-poly(butyl acrylate) and starch-graft-poly(butyl acrylate-co-styrene $75 / 25$ ) due to their severe brittleness. Table 4 summarizes the tensile strength and the strain at break ( $\varepsilon$ break) as a function of composition. In order to gain a better understanding of the mechanical

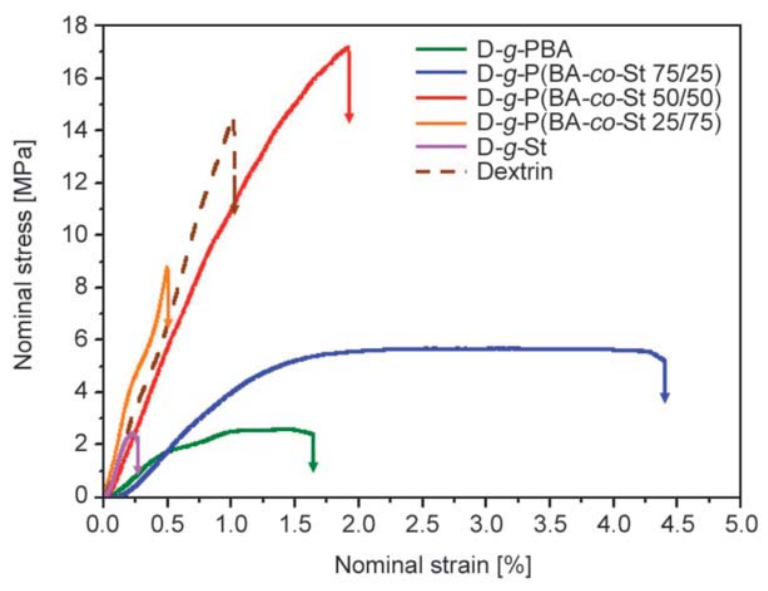

Figure 7. Nominal stress-strain curves of dextrin and dextrin-graft-poly(butyl acrylate-co-styrene) under uniaxial drawing at $20^{\circ} \mathrm{C}, 50 \% \mathrm{RH}$ at strain rate of $10^{-2} \mathrm{~s}^{-1}$. 
response of materials, the draw temperature $T_{\mathrm{d}}$ is considered by comparison to $T_{\mathrm{g} 1}$ and $T_{\mathrm{g} 2}$ of samples, corresponding respectively to the glass transition temperature of the dextrin/starch and poly(butyl acrylate-co-styrene) phases as defined previously.

Several interesting features may be drawn from Figure 7 and Table 4:

1. Regardless of composition, all materials are rather brittle, with a strain at break below $6 \%$. It is worth reminding that under these experimental conditions, the dextrin/starch phase is in the glassy state $\left(T_{\mathrm{g} 1}=55 \pm 3^{\circ} \mathrm{C}\right.$ as reported in Figure 6a) while the poly(butyl acrylate-co-styrene) phase can either be in the rubbery or in the glassy state depending on the composition of the grafted chains. These results show that even when the poly(butyl acrylate- $c o$-styrene) phase is in the rubbery state, materials exhibit poor drawability, suggesting that the overall mechanical behavior is rather controlled by the dextrin/starch phase in this case.

2. As long as the grafted chains are in a rubbery state, a slight influence of the grafted chain composition may be noticed. In particular, addition of styrene tends to increase the stiffness while increasing the butyl acrylate tends to improve the stretchability at the expense of the stress level. Note that an improvement of both tensile strength and strain at break is observed in the case of dextrin-graft-poly(butyl acrylate-co-styrene(50/50)) compared to pure dextrin which makes it potentially interesting for future applications.

3. When both starch/dextrin and poly(butyl acrylate-co-styrene) phases are in the glassy state, materials are extremely brittle as expected. Note that in this case, tensile strength values have to be taken with caution, because experiments were extremely difficult to perform.

4. The effect of molecular weight of the starch substrate on mechanical behavior is not obvious in the case of experiments conducted at $50 \% \mathrm{RH}$.

To better understand the role of each phase on the overall response of the material, uniaxial tensile tests have been performed at room temperature and $80 \% R H$. In that case, the dextrin/starch phase is in the rubbery state. Results are summarized in Table 4. Despite important standard deviation, as long as $T_{\mathrm{g} 2}$ is lower than $20^{\circ} \mathrm{C}$, i.e. both phases are in the rubbery state, a ductile behavior characterized by a strain at break of up to $100 \%$ is observed. By contrast, materials remain brittle as soon as $T_{\mathrm{g} 2}$ of the grafted chains
Table 4. Mechanical properties of dextrin-graft-poly(butyl acrylate- $c o$-styrene) under uniaxial tensile at $20^{\circ} \mathrm{C}$ at $50 \% R H$ and at $80 \% R H$.

\begin{tabular}{|c|c|c|c|}
\hline \multicolumn{4}{|c|}{$50 \% R H: T_{\mathrm{g} 1 \text { (dextrin/starch) }} \sim 55^{\circ} \mathrm{C}$} \\
\hline Material & \begin{tabular}{|c|}
$T_{\mathrm{g} 2 \text { (grafted chain) }}$ \\
{$\left[{ }^{\circ} \mathrm{C}\right]$}
\end{tabular} & $\begin{array}{c}\text { Strength } \\
\text { [MPa] }\end{array}$ & $\begin{array}{c}\varepsilon \text { break } \\
{[\%]}\end{array}$ \\
\hline D-g-PBA & \multirow{2}{*}{-44} & $2.1( \pm 0.4)$ & $1.8( \pm 0.5)$ \\
\hline S- $g$-PBA & & - & - \\
\hline D- $g$-P(BA-co-St 75/25) & \multirow{2}{*}{1} & $4.5( \pm 1.1)$ & $6.0( \pm 2.0)$ \\
\hline S- $g$-P(BA-co-St 75/25) & & - & - \\
\hline D- $g$-P(BA-co-St 50/50) & \multirow{2}{*}{24} & $17.7( \pm 3.7)$ & $2.4( \pm 0.7)$ \\
\hline S- $g$-P(BA-co-St 50/50) & & $15.3( \pm 4.9)$ & $3.9( \pm 1.2)$ \\
\hline D-g-P(BA-co-St 25/75) & \multirow{2}{*}{59} & $6.6( \pm 1.8)$ & $0.4( \pm 0.1)$ \\
\hline S- $g$-P(BA-co-St 25/75) & & $7.6( \pm 3.5)$ & $2.5( \pm 0.7)$ \\
\hline D-g-PS & \multirow{2}{*}{99} & $2.5( \pm 0.2)$ & $0.5( \pm 0.2)$ \\
\hline S-g-PS & & $4.9( \pm 1.0)$ & $2.6( \pm 0.8)$ \\
\hline \multicolumn{4}{|c|}{$80 \% R H: T_{\text {g1 (dextrin/starch) }} \sim 5^{\circ} \mathrm{C}$} \\
\hline Material & \begin{tabular}{|c|}
$T_{\mathrm{g} 2 \text { (grafted chain) }}$ \\
{$\left[{ }^{\circ} \mathrm{C}\right]$}
\end{tabular} & $\begin{array}{c}\text { Strength } \\
\text { [MPa] }\end{array}$ & $\begin{array}{c}\varepsilon \text { break } \\
{[\%]}\end{array}$ \\
\hline D-g-PBA & \multirow{2}{*}{-44} & $1.5( \pm 0.3)$ & $5.3( \pm 2.9)$ \\
\hline S-g-PBA & & - & - \\
\hline D-g-P(BA-co-St 75/25) & \multirow{2}{*}{1} & $2.6( \pm 1.0)$ & $97.0( \pm 80.0)$ \\
\hline S- $g$-P(BA-co-St 75/25) & & - & - \\
\hline D-g-P(BA-co-St 50/50) & \multirow{2}{*}{24} & $9.7( \pm 3.7)$ & $27.0( \pm 25.0)$ \\
\hline S- $g$-P(BA-co-St 50/50) & & $13.2( \pm 1.2)$ & $13.5( \pm 8.8)$ \\
\hline D- $g$-P(BA-co-St 25/75) & \multirow{2}{*}{59} & $8.7( \pm 1.6)$ & $1.5( \pm 0.3)$ \\
\hline S- $g$-P(BA-co-St 25/75) & & $9.8( \pm 2.8)$ & $0.7( \pm 0.2)$ \\
\hline D-g-PS & \multirow{2}{*}{99} & $7.7( \pm 1.6)$ & $1.3( \pm 0.2)$ \\
\hline S-g-PS & & $7.6( \pm 1.7)$ & $1.7( \pm 0.4)$ \\
\hline
\end{tabular}

is higher than the draw temperature. It seems that in this case, the poly(butyl acrylate-co-styrene) phase governs the mechanical behavior of the material.

To sum up, it seems possible to tune the mechanical properties by adjusting the water content in the dextrin phase and the grafted chain composition. In particular, as soon as one of the two phases is in the glassy state, this phase controls the mechanical response.

Similar trend is observed for starch-graft-poly(butyl acrylate-co-styrene) samples. However, in the case of ductile response (i.e. as soon as both phases are in the rubbery state), comparison of starch-based samples to dextrin-based ones reveals that the latter tend to display a higher strain at break, revealing the advantage of using dextrin when optimization of stretchability is targeted.

\section{Conclusions}

The synthesis of dextrin/starch-graft-poly(butyl acrylate-co-styrene) with different ratios of $\mathrm{BA} / \mathrm{St}$ was performed on two different starch substrates displaying 
various $M_{\mathrm{w}}$. During the grafting copolymerization, formation of long grafted chains $\left(M_{\mathrm{n}}=3.8 \cdot 10^{4}\right.$ $1.7 \cdot 10^{5} \mathrm{~g} / \mathrm{mol}$ ) is favored during the grafting copolymerization, resulting in a low degree of substitution $(D S<0.002)$. Thermal and structural analysis have shown that dextrin/starch-graft-poly(butyl acrylateco-styrene) materials are fully amorphous and confirm the formation of random poly(butyl acrylate-costyrene) copolymers. TEM microscopy observations have revealed that materials are organized into two co-continuous phases with domain sizes of several tens of nanometers. One phase is mainly composed of starch/dextrin macromolecules characterized by a $T_{\mathrm{g}}$ sensitive to moisture, while the second phase refers to the grafted polymer with a glass transition temperature dependent on the $\mathrm{BA} / \mathrm{St}$ ratio. Water uptake is governed by the amount of dextrin/starch inside the sample and diffusivity increases with the $\mathrm{BA} / \mathrm{St}$ ratio. Mechanical properties are highly dependent on the rubbery/glassy state of both phases. As soon as one of the phases is into the glassy state, it controls the overall behavior of the material. For a defined starch substrate, water content and grafted chain composition appear as the key parameters for tailoring the mechanical response of the materials. Interest in using dextrin instead of starch is highlighted in this study. Due to the lower viscosity of the reactive medium, the use of dextrin allows to graft synthetic polymers under less harsh conditions, while affording similar properties. In addition, the improved processability allows further the film elaboration over a wider range of compositions. Potential applications for these new materials could be as compatibilizers with various starch/synthetic hydrophobic polymers blends.

\section{References}

[1] Halley P. J., Avérous L.: Starch polymers: From genetic engineering to green applications. Elsevier, Amsterdam (2014).

https://doi.org/10.1016/C2009-0-64023-X

[2] Avérous L.: Biodegradable multiphase systems based on plasticized starch: A review. Journal of Macromolecular Science Part C: Polymer Reviews, 44, 231-274 (2007). https://doi.org/10.1081/MC-200029326

[3] Jiménez A., Fabra M. J., Talens P., Chiralt A.: Edible and biodegradable starch films: A review. Food and Bioprocess Technology, 5, 2058-2076 (2012). https://doi.org/10.1007/s11947-012-0835-4
[4] Mekonnen T., Mussone P., Khalil H., Bressler D.: Progress in bio-based plastics and plasticizing modifications. Journal of Materials Chemistry A, 1, 1337913398 (2013). https://doi.org/10.1039/C3TA12555F

[5] Chen Q., Yu H., Wang L., Abdin Z., Chen Y., Wang J., Zhou W., Yang R., Khan R., Zhang H., Chen X.: Recent progress in chemical modification of starch and its applications. RSC Advances, 5, 67459-67474 (2015). https://doi.org/10.1039/C5RA10849G

[6] Avérous L., Halley P. J.: Biocomposites based on plasticized starch. Biofuels Bioproducts and Biorefining, 3, 329-343 (2009).

https://doi.org/10.1002/bbb.135

[7] Hullemans S. H. D., Janssen F. H. P., Feil H.: The role of water during plasticization of native starches. Polymer, 39, 2043-2048 (1998).

https://doi.org/10.1016/S0032-3861(97)00301-7

[8] Rodriguez-Gonzalez P. J., Ramsay B. A., Favis B. D.: Rheological and thermal properties of thermoplastic starch with high glycerol content. Carbohydrate Polymers, 58, 139-147 (2004).

https://doi.org/10.1016/j.carbpol.2004.06.002

[9] Lourdin D., Coignard L., Bizot H., Colonna P.: Influence of equilibrium relative humidity and plasticizer concentration on the water content and glass transition of starch materials. Polymer, 38, 5401-5406 (1997). https://doi.org/10.1016/S0032-3861(97)00082-7

[10] Ma X., Yu J., Feng J.: Urea and formamide as a mixed plasticizer for thermoplastic starch. Polymer International, 53, 1780-1785 (2004).

https://doi.org/10.1002/pi.1580

[11] Sankri A., Arhaliass A., Dez I., Gaumont A. C., Grohens Y., Lourdin D., Pillin I., Roland-Sabaté A., Leroy E.: Thermoplastic starch plasticized by an ionic liquid. Carbohydrate Polymers, 82, 256-263 (2010). https://doi.org/10.1016/j.carbpol.2010.04.032

[12] Vargha V., Truter P.: Biodegradable polymers by reactive blending trans-esterification of thermoplastic starch with poly(vinyl acetate) and poly(vinyl acetate-co-butyl acrylate). European Polymer Journal, 41, 715-726 (2005).

https://doi.org/10.1016/j.eurpolymj.2004.10.044

[13] Wang X-L., Yang K-K., Wang Y-Z.: Properties of starch blends with biodegradable polymers. Journal of Macromolecular Science, 43, 385-409 (2003).

https://doi.org/10.1081/MC-120023911

[14] Shogren R. L.: Rapid preparation of starch esters by high temperature/pressure reaction. Carbohydrate Polymers, 52, 319-326 (2003). https://doi.org/10.1016/S0144-8617(02)00305-3

[15] Aburto J., Alric I., Borredon E.: Preparation of longchain esters of starch using fatty acid chlorides in the absence of an organic solvent. Starch/Stärke, 51, 132135 (1999).

https://doi.org/10.1002/(SICI)1521379X(199904)51:4<132::AID-STAR132>3.0.CO;2-Z 
[16] Meimoun J., Wiatz V., Saint-Loup R., Parcq J., Favrelle A., Bonnet F., Zinck P.: Modification of starch by graft copolymerization. Starch/Stärke, 70, 1600351-1600374 (2018). https://doi.org/10.1002/star.201600351

[17] Jyothi A. N.: Starch graft copolymers: Novel applications in industry. Composite Interfaces, 17, 165-174 (2010). https://doi.org/10.1163/092764410X490581

[18] Wang S., Xu J., Wang Q., Fan X., Yu Y., Wang P., Zhang Y., Yuan H., Cavaco-Paulo A.: Preparation and rheological properties of starch- $g$-poly(butyl acrylate) catalyzed by horseradish peroxidase. Process Biochemistry, 59, 104-110 (2017). https://doi.org/10.1016/j.procbio.2017.01.014

[19] Qu J., He L.: Synthesis and properties of silane-fluoroacrylate grafted starch. Carbohydrate Polymers, 98, 1056-1064 (2013).

https://doi.org/10.1016/j.carbpol.2013.07.015

[20] Meshram M. W., Patil V. V., Mhaske S. T., Thorat B. N.: Graft copolymers of starch and its application in textiles. Carbohydrate Polymers, 75, 71-78 (2009). https://doi.org/10.1016/j.carbpol.2008.06.012

[21] Mou J., Li X., Wang H., Fei G., Liu Q.: Preparation, characterization, and water resistance of cationic acetylated starch- $g$-poly(styrene-butyl acrylate) surfactantfree emulsion. Starch, 64, 826-834 (2012). https://doi.org/10.1002/star.201200041

[22] Cheng S., Xu J., Wu Y.: Preparation and characterization of oxidized starch-graft-poly(styrene-butyl acrylate) latex via emulsion polymerization. Journal of Polymer Engineering, 34, 611-616 (2014). https://doi.org/10.1515/polyeng-2014-0047

[23] Cheng S., Zhao W., Wu Y.: Optimization of synthesis and characterization of oxidized starch-graft-poly (styrene-butyl acrylate) latex for paper coating. Starch, 67, 493-501 (2015). https://doi.org/10.1002/star.201400265
[24] Qudsieh I. Y. M., Fakhru'I-Razi A., Muyibi S. A., Ahmad M. B., Rahman M. Z., Yunus W. M. Z. W.: Preparation and characterization of poly(methyl methacrylate) grafted sago starch using potassium persulfate as redox initiator. Journal of Applied Polymer Science, 94, 18911897 (2004). https://doi.org/10.1002/app.20883

[25] Vancso G., Snetivy D., Tomka I.: Structural changes during polystyrene orientation: A study of optical birefringence and wide angle X-ray scattering. Journal of Applied Polymer Science, 42, 1351-1359 (1991). https://doi.org/10.1002/app.1991.070420518

[26] Krimm S., Tobolsky A. V.: Quantitative X-ray studies of order in amorphous and crystalline polymers: Scattering from various polymers and a study of the glass transition in polystyrene and polymethyl methacrylate. Textile Research Journal, 21, 805-822 (1951). https://doi.org/10.1177/004051755102101106

[27] Miller R. L., Boyer R. F., Heijboer J.: X-ray scattering from amorphous acrylate and methacrylate polymers: Evidence of local order. Journal of Polymer Science: Polymer Physics Edition, 22, 2021-2041 (1984). https://doi.org/10.1002/pol.1984.180221203

[28] Floudas G., Štepánek P.: Structure and dynamics of poly( $n$-decyl methacrylate) below and above the glass transition. Macromolecules, 31, 6951-6957 (1998). https://doi.org/10.1021/ma9804601

[29] Fernandez-García M., Fernández-Sanz M., Madruga E. L., Cuervo-Rodriguez R., Hernández-Gordo V., Fernández-Monreal M. C.: Solvent effects on the free-radical copolymerization of styrene with butyl acrylate. I. Monomer reactivity ratios. Journal of Polymer Science Part A: Polymer Chemistry, 38, 60-67 (2000). https://doi.org/10.1002/(SICI)10990518(20000101)38:1<60::AID-POLA8 $>3.0 . C O ; 2-\mathrm{F}$

[30] Utracki L. A.: Polymer blends, Volume 11. Rapra, Shawbury (2000).

[31] Cheviron P., Gouanvé F., Espuche E.: Effect of silver nanoparticles' generation routes on the morphology, oxygen, and water transport properties of starch nanocomposite films. Journal of Nanoparticle Research, 17, 364/1-364/16 (2015). https://doi.org/10.1007/s11051-015-3173-4 